The Astrophysical Journal, 270:51-70, 1983 July 1

(C) 1983. The American Astronomical Society. All rights reserved. Printed in U.S.A.

\title{
STELLAR AND GASEOUS DYNAMICS OF TRIAXIAL GALAXIES
}

\author{
GEORGE LAKE $^{1,2}$ AND COLIN NoRMAN ${ }^{2,3}$ \\ Received 1982 August 2; accepted 1982 December 16
}

\begin{abstract}
We apply the results of our calculations of Hamiltonian systems to stars and gas in both normal and active galaxies. Gas motions are considered by applying Mel'nikov's method of calculating dissipative systems "close to Hamiltonian." This results in the "attraction" of gas to stable (nonintersecting) periodic orbits and "repulsion" from unstable period orbits. In the outer parts of galaxies, two disks may be populated, as is the case in NGC 2685. In the inner regions a tilted disk may be populated. In the central region, because the orbits are boxes, the streamlines will not close without shocks. The resultant effective viscosity will be large and provides enhanced fueling rates to any central massive object, thus producing an active galaxy. If this central massive object grows to half the core mass, the resultant symmetry restoration changes the character of the orbits and halts the enhanced fueling. We propose this as a possible evolutionary scenario for active galaxies.

We calculate the properties of orbits in three-dimensional nonaxisymmetric potentials which are chosen to approximate a triaxial elliptical galaxy. Two methods are used: (1) A class of potentials with singular behavior at the center is used to approximate the outer regions of the galaxy, i.e., $r \gg r_{c}$ where $r_{c}$ is a core radius. These potentials are structurally stable and have three exact isolating integrals. Reduced potentials are used to assess the existence and stability of periodic orbits and their associated families of orbits. For one particular subclass of these potentials, the equations of motion are fully integrated. (2) The potential at the center is expanded in harmonic plus quartic terms. A second integral of the motion is found for orbits near any 1-1-1 resonance. This integral is then used as a Liapunov function to test the stability of the $1-1-1$ orbits which are in general position (i.e., all the actions are nonzero, the most interesting family being that of elliptical orbits tilted with respect to the symmetry planes). Mathieu's equation is used to determine the stability of orbits with any of the actions equal to zero (elliptical orbits in a symmetry plane, linear orbits in the plane, normal mode or axial orbits).

We find as quite a general feature that families of orbits are associated with perturbations of stable fixed points (periodic orbits). Thus box orbits arise from the perturbation of the trivial fixed point at the origin (or alternatively, the perturbing of stable radial orbits), whereas tube orbits arise from the perturbation of stable elliptical orbits. At low energies there are three stable radial orbits, though two eventually become unstable at the bifurcation point of the elliptical orbits. All the orbits are box orbits at an energy below the bifurcation points. In both representations, at high energies, we find that only one radial orbit is stable to perturbations in any direction and only two of the three 1-1 elliptical orbits in the symmetry planes are stable to perpendicular oscillations. In the potential we use to describe the outer parts, the unstable elliptical orbit is not always the one in the plane perpendicular to the intermediate axis. At an intermediate energy we find the existence of a 1-1-1 elliptical orbit, tilted with respect to any of the symmetry planes. The stability of this orbit is dependent on energy and the values of the structure constants, but when stable it allows the construction of a stable, tilted disk. We propose that this is a plausible dynamical interpretation of the tilted disk seen in the galactic center. This implies that the central region of our Galaxy is triaxial.

Subject headings: galaxies: internal motions - galaxies: structure
\end{abstract}

\section{INTRODUCTION}

a) Purpose

This paper is concerned with the properties of motion of stars and gas in triaxial galaxies. Until recently, elliptical galaxies were assumed to be axisymmetric. Their flattening was considered to be a product of rotation, and their internal dispersions were thought to be isotropic. Recent observations of elliptical galaxies have shown

\footnotetext{
1 Physical Research Laboratory, Bell Laboratories.

2 Institute of Astronomy, Cambridge, England.

${ }^{3}$ Sterewacht, Huygens Laboratorium, Leiden, The Netherlands.
} 
that they are not flattened by rotation (Illingworth 1977) and that their isophotal profiles are not those of simple axisymmetric systems; twists are often evident in the outer parts (Evans 1952; King 1978). Classification schemes for galaxies are all based on a continuity of properties between spiral bulges and ellipticals. Certainly "barred spirals" are not axisymmetric, and even the bulge of M31 appears to be a triaxial figure (Stark 1977). $N$-body experiments of collapse have shown final state figures which are triaxial (Aarseth and Binney 1978; Miller 1978) and these figures can be long-lived (Miller and Smith 1980; Wilkinson and James 1982; van Albada 1982).

The modeling of elliptical galaxies as axisymmetric figures has considerable appeal due to its tractability (Prendergast and Tomer 1970; Wilson 1975; Lynden-Bell 1962a; Lake 1981). The dynamical state of a system is specified by the phase space distribution function, $f$ (i.e., the density of stars in both momentum and configuration space). Jeans's theorem that $f$ is a function of the integrals of the motion and the existence of the integrals $E$ and $L_{z}$, yields a problem solvable by Hartree-Fock methods when $f$ is specified or by inversion when the three-dimensional density is known (cf. Lake 1981 and references therein).

Recent numerical studies (Schwarzschild 1979; Richstone 1980) have shown the existence of two integrals in addition to the energy integral in potentials chosen to model the forms of elliptical galaxies. The existence of these integrals enabled Schwarzschild to construct an equilibrium model via a linear programming approach to discrete stellar dynamics. Our goal is to develop analytical methods which will provide a greater intuitive understanding of the integrals present in systems similar to that constructed by Schwarzschild and the relationship between these integrals and orbit families. In addition to this, we want to develop methods which will enable the calculation of new phenomena such as the evolution of a gaseous component, and which may eventually allow one to forego or at least diminish the computational demands of present approaches.

One additional goal of this paper is to connect the astrophysical problem of the dynamics of triaxial galaxies to the vast literature of modern dynamics and plasma physics. This leads to the unfortunate necessity of introducing some of the language which pervades these other fields. This is partly facilitated by $\S$ II wherein some of the new features of qualitative dynamics are described. We hope that introducing these unfamiliar concepts into the familiar domain of galactic dynamics will speed penetration into this new literature and avoid continual rediscovery of simple phenomena (for example, Mel'nikov's method, see $\S \mathrm{V}$ ).

\section{b) Introduction to Qualitative Dynamics}

In the last 20 years there has been a remarkable renaissance in classical physics. One of the principal issues has been: When do potentials admit isolating integrals (regular motion) and when is the ergodic hypothesis realized (irregular motion)? This seemingly simple issue, which has plagued and polarized physicists since the last century, is at long last being solved. Its consequences are far reaching, affecting even the very foundations of quantum theory (Einstein 1917) as well as dynamical problems in astrophysics and plasma physics.

For example, at the heart of quantum theory is the method of quantization itself. If the potential has isolating integrals (e.g., $E, J_{z}, J^{2}$ ), these integrals are quantized (Einstein's approach); whereas if the system is ergodic, then semiclassical quantization is employed. This difference is observed in molecular spectra (Percival 1973). In plasma physics, one may replace "orbit" with "field line" and ask about the stability of field configurations for plasma confinement (Rosenbluth et al. 1966). Here we are concerned about one of the oldest problems in this field: the dynamics of single particles in potentials designed to approximate galaxies (cf. Hénon and Heiles 1964).

The great advance of the last 20 years has been a shift in emphasis from individual particle orbits to the behavior of typical orbits, or the generic features of orbit families. This allows one to address whether "all," "none," "most," etc., orbits are ergodic or possess isolating integrals. In the case of nonaxisymmetric (or triaxial) potentials designed to match the photometric properties of galaxies, these potentials are "nearly integrable" (in the sense that "nearly all" orbits will behave as though they have three isolating integrals) according to various criteria for the onset of "the stochastic instability" (Chirikov 1979; Rosenbluth et al. 1966; Lake 1982). This feature is verified by numerical integration (Schwarzschild 1979).

The modern qualitative dynamics (cf. Arnold 1978) provides methods for the study of potentials "close to integrability" and systems "close to Hamiltonian" (i.e., those that involve dissipation). Key features in the former are the ideas of "structural stability" and bifurcation" (Chillingworth 1976). Bifurcations are the branchings of trajectories at critical points. In § IV we analyze a harmonic oscillator with potential perturbations which are quartic. In the innermost regions, the oscillations in each coordinate are linear and incommensurate. There are three periodic orbits, each along an axis. As the amplitudes are increased, resonances are possible which lead to destabilization of the radial orbits and the existence of elliptical orbits. These are the bifurcation points. Structural stability is the invariance of structural properties (for example, the nature of basic orbit families and the stability of periodic orbits) under finite perturbations of the initial Hamiltonian. All axisymmetric systems are structurally unstable; the single orbit family (tube orbits which encircle the symmetry axis, together with the limiting cases of radial orbits in the plane and unstable orbits "over the pole") becomes one of several orbit families in a nonaxisymmetric potential. A potential is structurally stable when perturbations do not create new bifurcations. 


\section{c) Outline}

With the goals enumerated in mind, in $\S$ II we analyze a particular class of potentials which possess three exact integrals of motion. This potential is chosen because of its resemblance to the potential used by Schwarzschild (1979) to produce an equilibrium model of a triaxial stellar figure. This potential is structurally stable. The structure of orbit families and the stability of periodic orbits are the same as in Schwarzschild's potential. In the Appendix, the expressions for the actions are derived and for a subclass of potentials the orbits are integrated.

In $\S$ III, we explore the properties of a potential based on an expansion about the core region. Such expansions have been shown by de Zeeuw and Merritt (1983) to be remarkably useful at predicting the structure of phase space at relatively large energies where the perturbation theory is not obviously valid. The reader who is more interested in new astronomical phenomena which may result from triaxial figures should proceed to $\S$ IV. Here we discuss new features of the central regions of galaxies, particularly the possibility of the formation of stable equilibrium tilted disks, the structure of the Spindle galaxy NGC 2685 (Shane 1980), and implications for enhanced fueling of gas into the central engine of active galaxies. Some of the material here has also been published elsewhere in a brief progress report of this research (Lake and Norman 1982).

\section{POTENTIALS WITH THREE EXACT INTEGRALS}

In this section, a class of potentials with three isolating integrals is introduced and used to explore the existence and stability of families of orbits. These potentials were discovered by Stäckel (1890) and further studied by Levi-Cevita (1904), Dell'Aqua (1908), Weinacht (1928), and Lynden-Bell (1962b).

The basic potential is:

$$
\Phi(r, \theta, \phi)=\xi(r)+\frac{\eta(\theta)}{r^{2}}+\frac{K(\phi)}{r^{2} \sin ^{2} \theta}
$$

where $\xi, \eta$, and $K$ are arbitrary functions. When Cartesian coordinates are used, it is with the usual conventions

$$
z=r \cos \theta, \quad x=r \sin \theta \cos \phi, \quad y=r \sin \theta \sin \phi .
$$

This potential has a form similar to that employed by Schwarzschild (1979) in his construction of a numerical model of a triaxial figure designed to match the photometric properties of the bulge of M31. Schwarzschild's potential has the form:

$$
\Phi=F(r)-G(r)\left(2-3 \sin ^{2} \theta\right)+H(r)\left(\sin ^{2} \theta \cos 2 \phi\right)
$$

where

$$
\begin{aligned}
& F(r)=-\frac{\log \left[r+\left(1+r^{2}\right)^{1 / 2}\right]}{r} \underset{r \rightarrow \infty}{\longrightarrow}-\frac{\ln (r)}{r}, \\
& G(r)=-\mathrm{cv}_{1} \cdot r^{2} \cdot\left(1+\mathrm{cv}_{2} \cdot r^{2}\right)^{-3 / 2} \underset{r \rightarrow \infty}{\longrightarrow}-\frac{\mathrm{cv}_{1}}{\left(\mathrm{cv}_{2}\right)^{3 / 2}} \frac{1}{r}, \\
& H(r)=-\mathrm{cw}_{1} \cdot r^{2} \cdot\left(1+\mathrm{cw}_{2} \cdot r^{2}\right)^{-3 / 2} \underset{r \rightarrow \infty}{\longrightarrow}-\frac{\mathrm{cw}_{1}}{\left(\mathrm{cw}_{2}\right)^{3 / 2}} \frac{1}{r},
\end{aligned}
$$

and $\mathrm{cv}_{1}, \mathrm{cv}_{2}, \mathrm{cw}_{1}, \mathrm{cw}_{2}$ are determined by the axis ratios of the triaxial figure (see deZeeuw and Merritt 1982).

The potential (1) admits three exact integrals of motion:

$$
\begin{aligned}
& H=\frac{1}{2} v^{2}-\Phi, \\
& I_{2}=\frac{1}{2} \varpi_{z}^{2}-K(\phi), \\
& I_{3}=\frac{1}{2} \varpi^{2}-\eta(\theta)-\frac{K(\phi)}{\sin ^{2} \theta} .
\end{aligned}
$$

$H$ is the Hamiltonian, where $v^{2}$ is the square of the total velocity. $I_{2}$ is a generalization of the square of the angular momentum in the $z$-direction $\left(\varpi_{z}^{2}\right)$. Finally, $I_{3}$ is a generalization of the square of the total angular momentum $\left(\varpi^{2}\right)$.

Using these three integrals, the separation of variables in the Hamilton-Jacobi equations yields:

$$
\begin{aligned}
& \frac{1}{2} P_{r}^{2}=\frac{1}{2} \dot{r}^{2}=H-\frac{I_{3}}{r^{2}}+\xi(r), \\
& \frac{1}{2} P_{\phi}^{2}=\frac{1}{2} r^{4} \sin ^{2} \theta \dot{\phi}^{2}=I_{2}+K(\phi), \\
& \frac{1}{2} P_{\theta}^{2}=\frac{1}{2} r^{4} \dot{\theta}^{2}=I_{3}-\frac{I_{2}}{\sin ^{2} \theta}+\eta(\theta) .
\end{aligned}
$$


The right-hand sides of these equations have the form of a constant and a reduced potential.

In order to exploit the similarity of the potentials (1) and (3), we use the particular form of the potential:

$$
\Phi(r, \theta, \phi)=\frac{G M}{b+\left(r^{2}+b^{2}\right)^{1 / 2}}+\frac{\alpha \sin ^{2} \theta}{r^{2}}+\frac{\beta(\cos 2 \phi-1)}{r^{2} \sin ^{2} \theta} .
$$

As in the potential used by Schwarzschild, the effect of each of the three pieces of this potential can be understood as follows. The first term is a purely spherical term and is chosen to be an isochrone potential. (For the discussion which immediately follows, any centrally concentrated potential could be used. Only when integrating the orbits does this choice become technically fortuitous.) The second term has the effect of lengthening (shortening) the $z$-axis relative to the $x$ and $y$ axes for values of $\alpha>0(<0)$. The third term lengthens the $x$-axis and shortens the $y$-axis equally for values of $\beta>0$. Taking $\beta<0$ simply reverses the roles of $x$ and $y$, so with no loss of generality we consider only $\beta>0$. Note that $\alpha>0$ does not necessarily imply that the $z$-axis is the longest axis. If $\beta>\alpha$, the $y$-axis becomes the shortest axis, with the $z$-axis being the intermediate one.

Choosing different values of $\alpha$ and $\beta$ to juggle the roles of short, intermediate, and long axes among $x, y$, and $z$ will yield different stability properties for the axis orbits along the various axes and the elliptical orbits perpendicular to them. With $\beta>0$, the elliptical orbit perpendicular to the $y$-axis is always unstable to perpendicular displacement and the other two elliptical orbits are stable. In this particular potential, the instability of the elliptical orbits perpendicular to the intermediate axis is not a generic feature.

Our particular choice of stretching and squashing axes is motivated by a desire to reproduce the stability properties of the elliptical orbits in Schwarzschild's model (Heiligman and Schwarzschild 1979). This is done by choosing $\alpha<0$, which compresses the $z$-axis. We then restrict $-\alpha>\beta>0$ which lengthens the $x$-axis and shortens the $y$-axis, but prevents the $y$-axis from becoming shorter than the $z$-axis.

All of this might seem rather artificial were it not for the ability of this potential to analytically reproduce so many other of the qualitative features seen by numerical integration in Schwarzschild's model. These features are best shown with the use of diagrams illustrating the reduced potentials. These are shown for the particular case $-\alpha>\beta>0$ in Figure 1. These diagrams are central to the understanding of this problem, and we suggest that the reader's (especially any casual reader's) time may be best spent studying these carefully. Note that in Figure 1(i), the scaling $b=\frac{1}{2}, G M=1$ has been used.

The simplest of these diagrams is the $\phi$ potential, as it is given by the function $K(\phi)$, here $\beta(\cos 2 \phi-1)$. This potential is that of a pendulum. At low energies the motion is librating, while at higher energies the motion is like that of the rotating pendulum. There are two points where there is no motion in $\phi$. The first is the potential minimum at $\phi=0$, which is stable to displacement, and orbits near this point librate back and forth in $\phi$. The second stationary point is at $\phi=\pi / 2$. This point is analogous to the pendulum teetering in the vertical position. Small perturbations of this orbit are unstable, leading to libration about $\phi=0$ or to rotation. This point is the separatrix (separating trajectory) dividing rotation and libration.

In the two angular coordinates there is always the possibility of rotation or libration. In the radial coordinate there is only a libration which may or may not include the center depending on the value of $I_{3}$ and thereby the existence of a centripetal barrier. There are then several basic orbit families:

$$
\begin{aligned}
\text { box orbits } \equiv & \text { libration in the angular coordinates, } \\
& \text { libration in the radial coordinate including the origin; } \\
\text { shell orbits } \equiv & \text { libration in the angular coordinates, } \\
& \text { libration in the radial coordinate excluding the origin; }
\end{aligned}
$$

The combinatorially minded reader will have noticed that there are no orbit families with either (1) rotation in both angular coordinates or (2) rotation in either angular coordinate combined with a libration in the radial coordinate which includes the origin. The reason is that at $\theta=0, \phi$ is undefined; and at $r=0$, both $\theta$ and $\phi$ are undefined. Rotation cannot occur in both angles because at $\theta=0$ the particle cannot preserve any sense of which direction it was going in $\phi$. Stated in this manner, it appears to be a property of the particular coordinate system or potential that was chosen. This is not so; it is quite a general topological property (cf. Berry 1978; Chillingworth 1976).

We now turn to the use of the reduced potential diagrams as a means of finding the stability of axial and elliptical orbits, the correspondence of these stability properties to the existence of orbit families and the specific range of values of $H, I_{2}$, and $I_{3}$ which delineate the orbit families. Rotation about the long axis occurs for $I_{2}>0$ and is accompanied by stable libration in $\theta$ and $r$. When the amplitudes of the $\theta$ and $r$ oscillations are zero, the orbit is closed and roughly elliptical. Increasing the values of $\mathrm{H}$ or $\mathrm{I}_{3}$ from this minimum value yields the family of tubes 

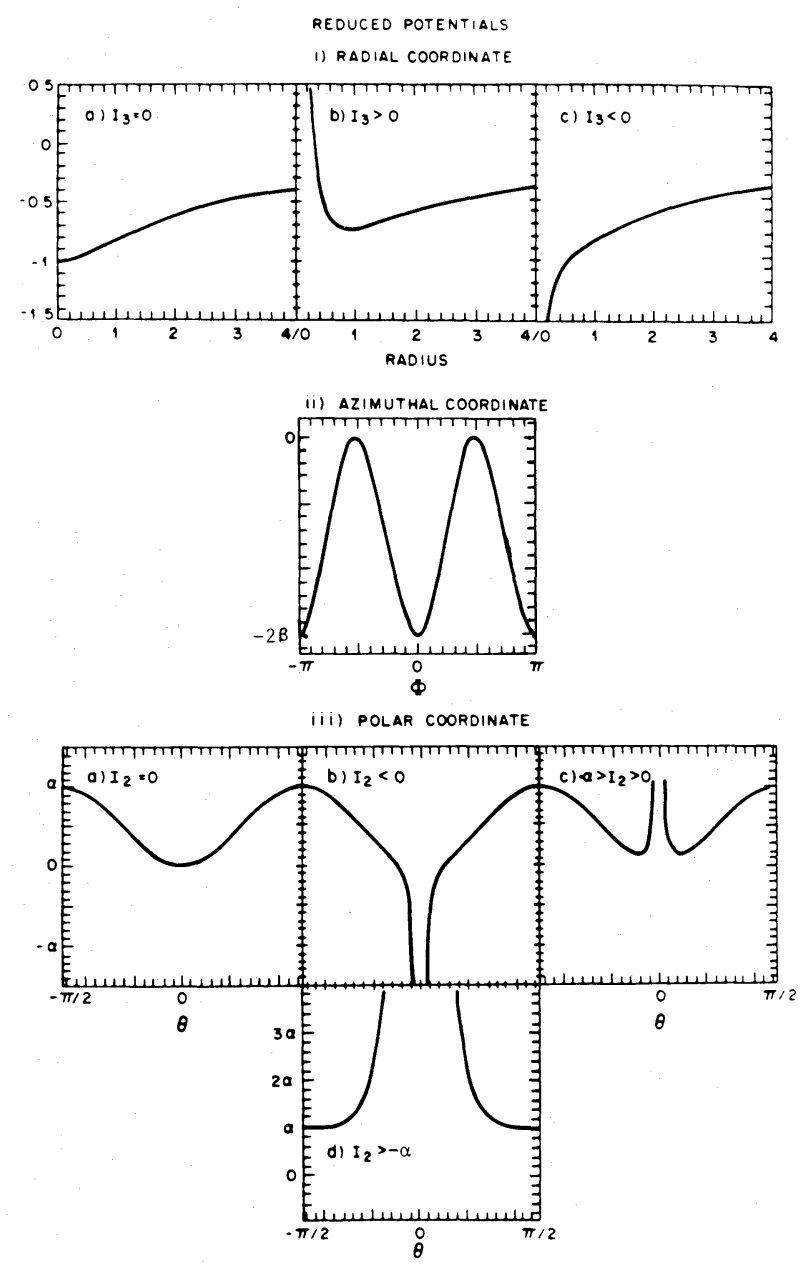

FIG. 1.-Reduced potentials from eqs. (6) and (7). These potentials show the types of motion possible in each of the three separated coordinates.

about the long axis. $I_{2}<0$ traps the particle into libration near the $x$-axis which for specific values of $I_{2}$ and $I_{3}$ produces $x$-tubes $\left(I_{3}>-\alpha-I_{2}, I_{2}<0\right)$, shell orbits $\left(0<I_{3}<-\alpha-I_{2}, I_{2}>0\right)$ and box orbits $\left(I_{2}<0, I_{3}<0\right)$. Note that $I_{2}=0$ is indeed the separating trajectory for those families of orbits. As a consequence it has no associated orbit family; i.e., there are no $y$-tubes (tubes encircling the intermediate axis). There is one other interesting feature to be seen in the reduced potentials. Note in Figure 1.3a that when $-\alpha>I_{2}>0$, the circulating orbit may be tilted out of the plane and is stable to perturbation.

We leave the derivation of the stability properties of axial orbits as an exercise for the industrious reader. The results are stated in Table 1, a summary of qualitative features of the orbits. Note that the stability of the axial orbits is the same as in Schwarzschild's model (Goodman and Schwarzschild 1980).

We conclude this section with a few remarks about the structural stability (Chillingworth 1976) of the potential (1). Structural stability is concerned with whether or not the qualitative features of the basic phase space trajectories are sensitive to small perturbations. Structural instability occurs when small changes lead to new bifurcations and thus to new families of orbits. As an example, consider the potential (1) with $K(\phi)=0$. These axisymmetric potentials are examples of structurally unstable systems. In these potentials there are only unstable axial orbits and tube orbits which circulate about the axis of symmetry. Even a small value of the parameter $\beta$ results in a different structure of the orbits.

Verhulst (1979) has shown that the potential (1) with $\beta=0$ is structurally stable when expanded about circular orbits (i.e., when only the reduced potentials in $r$ and $\theta$ are examined). The constancy of the qualitative features between the potential (1) and the form (3) used by Schwarzschild leads us to conclude that the potential is structurally stable. 
TABLE 1

Summary OF Qualitative Features of THE Model $-\alpha>\beta>0$

A. Stability of Elliptical Orbits to Perpendicular Displacement Circulating about and Perturbed in:

Short axis: stable.

Intermediate axis: unstable.

Long axis: stable.

B. Stability of Radial Orbits to Perpendicular Displacement

An orbit along the short axis is:

Unstable to all perpendicular displacements.

An orbit along the intermediate axis is:

Stable to displacement along the short axis, but unstable to displacement along the long axis.

An orbit along the long axis is:

Stable to all perpendicular displacements.

C. Orbit Families and Corresponding Ranges of $H, I_{2}, I_{3}$

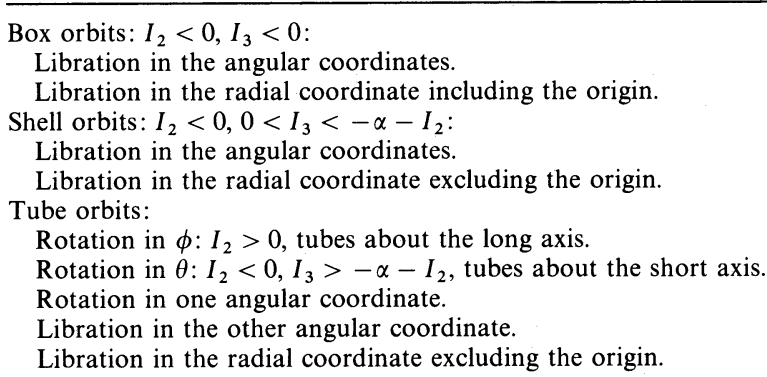

III. DYNAMICS OF THE CENTRAL REGION OF TRIAXIAL SYSTEMS

a) The Potential and the Resulting Integrals of Motion for the 1-1-1 Resonance

The singular behavior at the origin of the potential in the previous section makes it a poor approximation of a triaxial galaxy in the central region of a few core radii. To circumvent this problem and to gain further insight into the properties of orbit families, in this section we explore a potential based on perturbations of harmonic oscillators. Only the nonrotating case is considered here. Work on the rotating case is in progress (see de Zeeuw 1982).

The Hamiltonian is given by the expression:

$$
H=\frac{1}{2} \dot{x}^{2}+\frac{1}{2} \dot{y}^{2}+\frac{1}{2} \dot{z}^{2}+\frac{1}{2} x^{2}+\frac{1}{2} b y^{2}+\frac{1}{2} f z^{2}-\frac{c x^{2} y^{2}}{2}-\frac{g x^{2} z^{2}}{2}-\frac{h y^{2} z^{2}}{2}-\frac{d}{4} x^{4}-\frac{e}{4} y^{4}-\frac{k}{4} z^{4} .
$$

The time variable has been rescaled such that the coefficient of $x^{2}$ is $\frac{1}{2}$ rather than $a / 2$. This is the Birkhoff normal form for the calculation of the integrals, though later calculations will include the coefficient $a$ to avoid tedious rescaling. This is the generalization to three dimensions of the two-dimensional potential investigated by de Zeeuw and Merritt (1983), who compared their results with results of numerical integrations of planar orbits in Schwarzschild's model. The three-dimensional Hamiltonian (8) and its modified form including rotation are under investigation by de Zeeuw. Our treatment is far less detailed than his, but we introduce a few new calculational tools and examine a few phenomena in greater detail.

In particular, we are interested in solutions near the resonances where the oscillation frequencies in the three coordinates are equal, the 1-1-1 resonance. Verhulst (1979) has shown in the two dimensional case that the 3:1 and 1:1 resonance are the lowest order resonances. Hamiltonians of the type (8) chosen to represent galaxies will not be sufficiently flattened to admit 3:1 resonance, hence the suspicion (justified by orbit integrations) is that the 1:1 resonance is the most important. De Zeeuw and Merritt (1983) have shown that the bulk of Schwarzschild's potential (1979) lies in the 1:1 resonance zone and the structure of phase space is well represented by this resonance.

By manipulating the equations of motion and using standard methods of averaging (cf. van der Burgh 1976; Verhulst 1979) the following approximate integrals can be found:

$$
\begin{gathered}
H_{0}=I_{1}+I_{2}+I_{3}, \\
H_{1}=c I_{1} I_{2} \cos ^{2}\left(\theta_{1}-\theta_{2}\right)+g I_{1} I_{3} \cos ^{2}\left(\theta_{1}-\theta_{3}\right)+h I_{2} I_{3} \cos ^{2}\left(\theta_{2}-\theta_{3}\right) \\
+3\left[d I_{1}{ }^{2}+e I_{2}{ }^{2}+k I_{3}{ }^{2}+(1-b) I_{2}+(1-f) I_{3}\right],
\end{gathered}
$$


where

$$
\begin{aligned}
& I_{1}=\frac{1}{2} \dot{x}^{2}+\frac{1}{2} x^{2} \equiv \frac{1}{2} A_{1}{ }^{2}, \\
& I_{2}=\frac{1}{2} \dot{y}^{2}+\frac{1}{2} y^{2} \equiv \frac{1}{2} A_{2}{ }^{2}, \\
& I_{3}=\frac{1}{2} \dot{z}^{2}+\frac{1}{2} z^{2} \equiv \frac{1}{2} A_{3}{ }^{2},
\end{aligned}
$$

and

$$
\begin{aligned}
& \left(\theta_{1}-\theta_{2}\right) \equiv \text { difference in phase in the oscillation of } x \text { and } y, \\
& \left(\theta_{2}-\theta_{3}\right) \equiv \text { difference in phase in the oscillation of } y \text { and } z \text {. }
\end{aligned}
$$

\section{b) A Simple Galerkin Method for Approximate Periodic Solutions}

In this section, the Galerkin method (Galerkin 1915; cf. Hall and Watt 1976) is used to solve for the resonant orbits to first order. The Galerkin method is one that specifies the frequency of periodic motion and then approximates the motion by successive Fourier components. Solving for a periodic orbit by specifying the initial conditions generally leads to the problem of small divisors (Siegel 1954). To circumvent this scourge of perturbation theory, most modern schemes focus on orbits with constant period, rather than energy or amplitude. This is one of the principles of the KAM theory (Kolmogorov 1954; Arnold 1963; Moser 1962).

Binney (1981) has analyzed the motion of particles in a logarithmic potential with a softened core and found that the orbits have discrete frequencies, indicative of the existence of a complete set of actions or integrals in involution. He further finds that the amplitude of the higher harmonics diminishes rather rapidly - a further justification for lower order expansions. Helleman and Bountis (1977) have used a first order Galerkin method to find the regions of strong overlap of resonances (a technique for predicting stochasticity) in the Hénon-Heiles (1964) potential. For an example of its application to higher orders, see Urabe, Yanagiwara, and Shinohara (1960) and Urabe (1966) for their treatment of the van der Pol equation.

This method will have neither a large domain of validity nor high accuracy, being yet another approximation in addition to the expansion of the potential. The method is not an adequate substitute for explicit orbit calculation. Unfortunately, explicit orbit calculation presupposes values of the structure constants and may miss new features by unsuitable choices. In uncharted waters, even a poor map would be of great aid. The approximate solutions for the orbits together with the integrals of motion (9) employed as constants of the motion or as Liapunov functions will guide us to at least one new dynamical feature: a stable tilted disk in the inner regions.

The simplest orbits one can start with are the axial orbits (henceforth Type I orbits). The equation of moving along the $y$-axis is:

We approximate the solution by

$$
\ddot{y}=-b y+e y^{3} .
$$

and find from the first order terms

$$
y=B_{1} \sin \omega t,
$$

$$
\text { and find from the first order terms }
$$

$$
B_{1}^{2}=\frac{4}{3} \frac{\left(b-\omega^{2}\right)}{e} .
$$

Using the same scaling of de Zeeuw and Merritt (1983) this corresponds to an energy

$$
E_{1}=\frac{2}{3} b \frac{\left(b-\omega^{2}\right)}{e} .
$$

This result can be compared to the exact relation between amplitude and frequency:

$$
\omega=\frac{2 \pi}{T}=2 \pi\left\{4 \int_{0}^{B} \frac{d y}{[2(E-y)]^{1 / 2}}\right\}^{-1}=\frac{\pi}{2}\left(\frac{e}{2}\right)^{1 / 2} \frac{\left(2 b / e-B^{2}\right)^{1 / 2}}{K\left[B^{2} /\left(2 b / e-B^{2}\right)\right]},
$$

where

$$
K(m)=\int_{0}^{\pi / 2}\left(1-m \sin ^{2} \theta\right)^{-1 / 2} d \theta
$$

is the complete elliptic integral of the first kind and the energy $E=b B^{2} / 2-e B^{4} / 4$. The approximate form (14) and the exact relation (15) are plotted in Figure 2.

We next consider orbits in one of the symmetry planes. If we take the $x-y$ plane, the equations of motion are:

$$
\begin{aligned}
& \ddot{x}=-a x+c x y^{2}+d x^{3}, \\
& \ddot{y}=-b y+c x^{2} y+e y^{3} .
\end{aligned}
$$




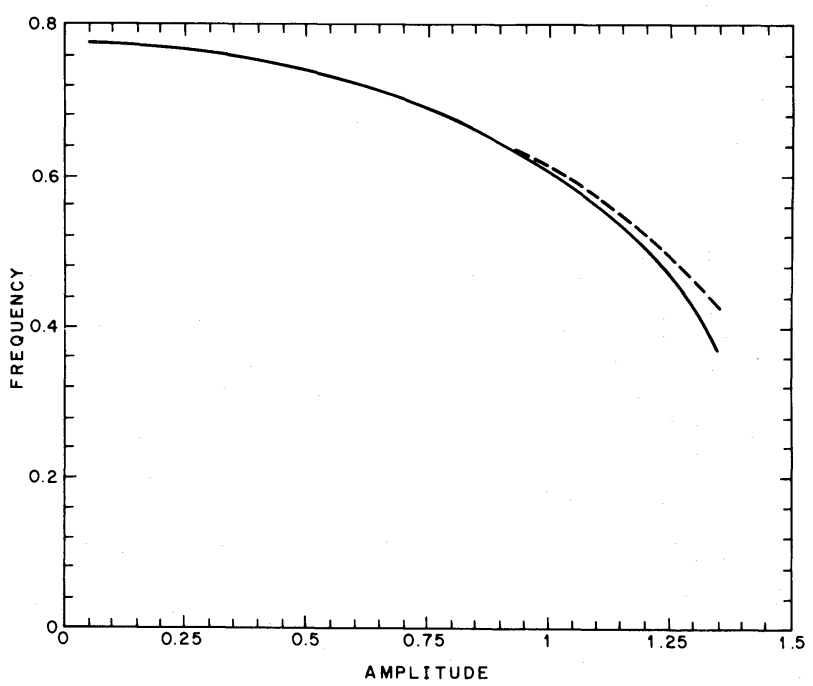

FIG. 2.-Amplitude versus frequency for the potential $\frac{1}{2}(0.6039) y^{2}-\frac{1}{4}(0.3068) y^{4}$. Solid line, the exact calculation (eq. [15]); dashed line, the Galerkin approximation (eq. [13]).

We can consider two types of resonances, the elliptical orbit with $x$ and $y 90^{\circ}$ out of phase (Type III orbits) and the linear orbit with $x$ and $y$ in phase (Type II orbits).

To solve for the Type III orbits, let

$$
\begin{aligned}
& x(t)=A \cos \omega t, \\
& y(t)=B \sin \omega t .
\end{aligned}
$$

Retaining only first order terms yields:

$$
\begin{aligned}
& A^{2}=4\left[\frac{\omega^{2}(3 e-c)-(3 e a-b c)}{c^{2}-9 e d}\right], \\
& B^{2}=4\left[\frac{\omega^{2}(3 d-c)+(c a-3 d b)}{c^{2}-9 e d}\right] .
\end{aligned}
$$

Calculating the maximum frequency for which $A^{2}>0, B^{2}>0$ yields the point of bifurcation of the elliptical orbit. For the potential used by Schwarzschild, this condition is:

$$
\omega^{2}=\frac{3 a-b c}{c-3 e}=0.38
$$

corresponding to an amplitude of $B^{2}=0.98$ and a scaled energy of 0.18 . This energy differs by approximately $20 \%$ from the value of 0.23 found by Schwarzschild.

The same procedure for the Type II orbit predicts an amplitude $B^{2}=1.8$, dangerously close to the amplitude 1.97 where the oscillations are unbounded. The Type II orbit has not been found by Schwarzschild.

Finally, we consider fully three-dimensional 1-1-1 periodic orbits. There are three different types of three-dimensional elliptical orbits (henceforth, Type IV orbits): one with each of the three coordinates out of phase with the other two. There are also three-dimensional linear orbits with all three oscillations in phase (henceforth, Type $\mathrm{V}$ orbits). The solution for the amplitudes of these orbits in our Galerkin approximation is found by solving the simple matrix equation

$$
\left[\begin{array}{ccc}
\frac{3 d}{4} & \left(\frac{1}{2}+\frac{1}{4} \cos \psi_{1}\right) c & {\left[\frac{1}{2}+\frac{1}{4} \cos \left(\psi_{1}+\psi_{2}\right)\right] g} \\
\left(\frac{1}{2}+\frac{1}{4} \cos \psi_{1}\right) c & \frac{3 e}{4} & \left(\frac{1}{2}+\frac{1}{4} \cos \psi_{2}\right) h \\
{\left[\frac{1}{2}+\frac{1}{4} \cos \left(\psi_{1}+\psi_{2}\right)\right] g} & \left(\frac{1}{2}+\frac{1}{4} \cos \psi_{2}\right) h & \frac{3 k}{4}
\end{array}\right]\left[\begin{array}{l}
A^{2} \\
B^{2} \\
C^{2}
\end{array}\right]=\left[\begin{array}{l}
a-\omega^{2} \\
b-\omega^{2} \\
f-\omega^{2}
\end{array}\right],
$$

where $A, B$, and $C$ are the amplitudes of the $x, y$, and $z$ oscillations, respectively, and $\psi_{1} \equiv 2\left(\theta_{1}-\theta_{2}\right), \psi_{2} \equiv 2\left(\theta_{2}-\theta_{3}\right)$ (see eqs. [9]). The four cases are obtained by solving (20) for $\psi_{1}=0, \psi_{2}=0$ (Type V orbits); $\psi_{1}=\pi, \psi_{2}=0$ 
(Type IV orbit, $x$ out of phase); $\psi_{1}=0, \psi_{2}=\pi$ (Type IV orbit, $z$ out of phase); and finally $\psi_{1}=\pi, \psi_{2}=\pi$ (Type IV orbit, $y$ out of phase).

\section{c) Stability of the Periodic Orbits}

In $\S$ II, the relation between stable periodic orbits and orbit families was explored. The potentials which describe triaxial galaxies are dominated by 1-1-1 resonances in the inner regions. Understanding the stability of the periodic orbits will provide insight into the dominant families of orbits.

Surprisingly, the orbits with all three amplitudes $(x, y, z)$ nonzero are those whose stability is most easily determined. (The procedure is analogous to that used by Verhulst (1979) for two-dimensional orbits.) These are the orbits in "general position." To find the stability of these orbits, the second integral (9b) is expanded in the neighborhood of the orbit, keeping only the quadratic terms. The first integral (9a) is held at constant value, and the definiteness of the quadratic form is examined. If the change in this form has the same sign independent of direction, it is definite and the orbit is stable. If the change is positive in some directions and negative in other directions, the form is indefinite and the orbit is unstable. This amounts to using the second integral as a Liapunov function in the fourdimensional space resulting from the resonance condition and the assumption of constant energy. The Liapunov stability in this space is equivalent to orbital stability in the full six-dimensional space. We recall that Liapunov stability is a synchronous stability where two particles started from nearby points in phase space remain close. Orbital stability is an asynchronous stability where only the trajectories are required to be close together, though the rate at which the orbits are traversed may change.

When the integral $(9 \mathrm{~b})$ is expanded, the first order terms can be shown to vanish directly from the equations of motion. Eliminating $A_{2}$ by the condition that the first integral $H_{0}$ is constant, the second order terms are:

$$
\begin{aligned}
& \delta^{2} I=\left(\delta A_{1}\right)^{2}\left[-\frac{3 e E}{2}+(b-a)+c E\left(1+\frac{1}{2} \cos \psi_{1}\right)+12 A_{1}{ }^{2}\left[\frac{3(d+e)}{16}-\frac{c}{4}\left(1+\frac{1}{2} \cos \psi_{1}\right)\right]\right. \\
& \left.+A_{3}{ }^{2}\left\{\frac{g}{2}\left[1+\frac{1}{2} \cos \left(\psi_{1}+\psi_{2}\right)\right]-\frac{c}{2}\left(1+\frac{1}{2} \cos \psi_{1}\right)-\frac{h}{2}\left(1+\frac{1}{2} \cos \psi_{2}\right)+\frac{3 e}{4}\right\}\right] \\
& \left.+\delta A_{1} \delta A_{3} \llbracket 2 A_{1} A_{3}\left\{\frac{g}{2}\left[1+\frac{1}{2} \cos \left(\psi_{1}+\psi_{2}\right)\right]-\frac{c}{2}\left(1+\frac{1}{2} \cos \psi_{1}\right)-\frac{h}{2}\left(1+\frac{1}{2} \cos \psi_{2}\right)+\frac{3 e}{4}\right\}\right] \\
& +\left(\delta A_{3}\right)^{2}\left[-\frac{3 e E}{2}+(b-f)+h E\left(1+\frac{1}{2} \cos \psi_{1}\right)+12 A_{3}{ }^{2}\left[\frac{3(e+k)}{16}-\frac{h}{4}\left(1+\frac{1}{2} \cos \psi_{2}\right)\right]\right. \\
& \left.+A_{1}^{2}\left\{\frac{g}{2}\left[1+\cos \left(\psi_{1}+\psi_{2}\right)\right]-\frac{c}{2}\left(1+\frac{1}{2} \cos \psi_{1}\right)-\frac{h}{2}\left(1+\frac{1}{2} \cos \psi_{2}\right)+\frac{3 e}{4}\right\}\right] \mid \\
& +\left(\delta \psi_{1}\right)^{2}\left[-\frac{c}{4} A_{1}{ }^{2} E \cos \psi_{1}+\frac{c}{8} A_{1}{ }^{2} \cos \psi_{1}+\frac{c}{8} A_{1}{ }^{2} A_{3}{ }^{2} \cos \psi_{1}-\frac{g}{8} A_{1}{ }^{2} A_{3}{ }^{2} \cos \left(\psi_{1}+\psi_{2}\right)\right] \\
& +\delta \psi_{1} \delta \psi_{2}\left[-\frac{g}{4} A_{1}{ }^{2} A_{3}{ }^{2} \cos \left(\psi_{1}+\psi_{2}\right)\right]+\left(\delta \psi_{2}\right)^{2}\left[-\frac{h}{4} A_{3}{ }^{2} E \cos \psi_{2}+\frac{h}{8} A_{3}{ }^{4} \cos \psi_{2}\right. \\
& \left.+\frac{h}{8} A_{1}{ }^{2} A_{3}{ }^{2} \cos \psi_{2}-\frac{g}{8} A_{1}{ }^{2} A_{3}{ }^{2} \cos \left(\psi_{1}+\psi_{2}\right)\right]+0\left(\delta \psi_{1} \delta A_{1}+\delta \psi_{2} \delta A_{3}+\delta \psi_{1} \delta H_{3}+\delta \psi_{2} \delta A_{1}\right) \text {. }
\end{aligned}
$$

As an example of the stability conditions obtained for a particular orbit, we use the Type IV orbit with $\psi_{1}=\pi, \psi_{2}=\pi$. Equation (21) becomes

$$
\begin{aligned}
& \delta^{2} I=\delta A_{1}{ }^{2}\left\{A_{1}{ }^{2}\left[\frac{3}{2}(d+e)-c\right]\right\}+\delta A_{1} \delta A_{3}\left\{A_{1} A_{3}\left[\frac{3(e+8)-(c+h)}{2}\right]\right\}+\delta A_{3}{ }^{2}\left\{A_{3}{ }^{2}\left[\frac{3(e+k)}{2}-h\right]\right\}
\end{aligned}
$$

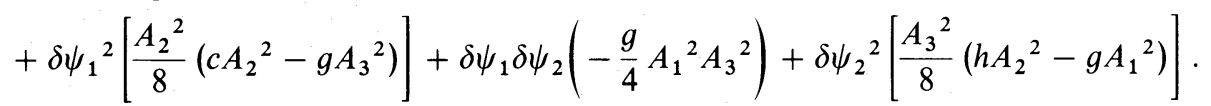

This yields the stability conditions:

$$
\begin{gathered}
{[6(d+e)-4 c]>\frac{[3(e+g)-(c+h)]^{2}}{6(e+k)-4 h}>0,} \\
c A_{2}{ }^{2}-g{A_{3}}^{2}>\frac{g}{\left(h A_{2}{ }^{2}-g A_{1}{ }^{2}\right)}>0 ;
\end{gathered}
$$

or the above with all inequality signs reversed. 
The stability of the other orbit types (I-III) must be analyzed directly by linearization about the orbit. As an example, we consider perturbations of the $y$ axial orbit in $x$. We approximate the $y$-motion as:

$$
y=B \sin \omega t,
$$

where

$$
B^{2}=\frac{4}{3} \frac{\left(b-\omega^{2}\right)}{e}
$$

The equation of motion for $x$ is:

$$
\ddot{x}=\left(-a+\frac{c B^{2}}{2}-\frac{c B^{2}}{2} \cos 2 \omega t\right) x,
$$

which is Mathieu's equation. The criterion for instability is:

$$
-q-q^{2} / 8<\alpha-1<q-q^{2} / 2,
$$

where

$$
\begin{aligned}
& q=\frac{c B^{2}}{4 \omega^{2}}, \\
& \alpha=\frac{1}{\omega^{2}}\left(a-\frac{c B^{2}}{2}\right) .
\end{aligned}
$$

At the onset of instability, the term $q^{2}$ is quite small and the orbit enters the unstable zone "from the left" in equation (27). Without the $q^{2}$ term this equation is equivalent to the conditions for the maximum frequency elliptical orbit (see $\S \mathrm{III} b$ ). The axial orbit becomes unstable at the point where the elliptical orbit bifurcates, which is known to be a general phenomenon (cf. Verhulst 1979). The stability of these orbits has been greatly studied (Binney 1978, 1981; Heilegman and Schwarzschild 1979; Martinet, in preparation), so we omit any further discussion here.

\section{NEW ASTROPHYSICAL PHENOMENA ASSOCIATED WITH TRIAXIALITY}

\section{a) Gas Motions. I. Spindle Structure}

\section{i) Observations and Interpretations}

One of the first interesting consequences of considering the dynamics of triaxial figures was the appearance of two families of tube orbits which circulated about two perpendicular axes (Schwarzschild 1979). The first existing system which comes to mind is NGC 2685, often referred to as the Spindle galaxy. An optical photograph of this system (cf. Shane 1980) shows an elongated system with a "helix-like" structure of dust and stellar associations wrapped around it like bands on a cigar. Detailed H I maps (Shane 1980) show the "helix-like" structure quite clearly and further demonstrate the existence of another ring which is on the major axis. In the inner region of the Spindle, within $10^{\prime \prime}$ of the nucleus (a distance of $50 \mathrm{pc}$ for a distance of $10 \mathrm{Mpc}$ to the galaxy), Ulrich (1975) and Schechter and Gunn (1978) find ionized gas with a sense of rotation similar to that of the helix and a projected velocity at $10^{\prime \prime}$ from the nucleus of $130 \mathrm{~km} \mathrm{~s}^{-1}$. The existence of the gas in two separate planes is impossible to reconcile with a simple axisymmetric system, as the orbits over the pole are always unstable.

Before examining the fate of gas in a triaxial galaxy, we consider the origin of the gas. Such an exotic system has attracted numerous even more exotic theories of origin. The existence of many pairs of interacting galaxies (Arp 1967), and the large numbers of dwarf companions possessed by spiral galaxies (Holmberg 1969) and their likelihood of being dragged inward by dynamical friction in the halo (Ostriker and Turner 1979), support Toomre's (1977) suggestion that we are seeing the late stages of accretion of a gas-rich dwarf galaxy. Further support can be found from the actual system parameters. An accreted dwarf galaxy will be disrupted when its density is roughly equal to the mean density of the parent galaxy inside the radius of its orbit, i.e.,

$$
\frac{M_{p}\left(r_{\text {break }}\right)}{\left(r_{\text {break }}\right)^{3}} \sim \frac{M_{\text {dwarf }}}{\left(r_{\text {dwarf }}\right)^{3}} .
$$

Here, $M_{p}\left(r_{\text {break }}\right)$ is the mass of the parent galaxy inside the breakup radius $\left(r_{\text {break }}\right)$, and $M_{\mathrm{dwarf}}$ and $r_{\mathrm{dwarf}}$ are the mass and characteristic radius of the dwarf galaxy.

Shane's (1980) observations determine the H I masses of the two structures, their outer radii, and the mass of the parent galaxy. These parameters are given in Table $2 \mathrm{~A}$, together with the derived parameters for the dwarf galaxy which may have been disrupted. A value of $\left(M_{\mathrm{H}} / M_{\text {total }}\right)=0.5$ has been assumed. These masses and radii can be 
TABLE $2^{\mathrm{a}}$

A. DWarf Properties Inferred from Equation (44)

\begin{tabular}{|c|c|c|c|c|}
\hline $\begin{array}{l}r_{\text {break }} \\
(\mathrm{kpc})\end{array}$ & $\begin{array}{c}M_{\text {parent }} \\
\left(10^{9} M_{\odot}\right)\end{array}$ & $\begin{array}{c}M_{\text {dwarf }} \\
\left(10^{9} M_{\odot}\right)\end{array}$ & $\begin{array}{l}r_{\mathrm{dwarf}} \\
(\mathrm{kpc})\end{array}$ & $\begin{array}{c}V_{c} \\
\left(\mathrm{~km} \mathrm{~s}^{-1}\right)\end{array}$ \\
\hline $3 \ldots \ldots \ldots \ldots$ & 20 & 1.0 & 1.1 & 63 \\
\hline $6 \ldots \ldots \ldots \ldots \ldots \ldots$ & 30 & 1.0 & 1.9 & 48 \\
\hline $12 \ldots \ldots \ldots \ldots \ldots \ldots$ & 60 & 2.0 & 3.9 & 48 \\
\hline
\end{tabular}

B. Properties of Some Known Dwarf Galaxies

\begin{tabular}{ccccc}
\hline \hline Name & $\begin{array}{c}\text { Mass } \\
\left(10^{9} M_{\odot}\right)\end{array}$ & $\begin{array}{c}R_{\text {HOLM }} \\
(\mathrm{kpc})\end{array}$ & $\begin{array}{c}V_{c} \\
\left(\mathrm{~km} \mathrm{~s}^{-1}\right)\end{array}$ & Ref $^{\mathrm{b}}$ \\
\hline SMC $\ldots \ldots \ldots \ldots \ldots$ & 1.4 & 4.7 & 41 & $\mathrm{~T}$ \\
N3109 $\ldots \ldots \ldots \ldots \ldots$ & 6 & 5.0 & 53 & $\mathrm{~T}$ \\
N6822 $\ldots \ldots \ldots \ldots \ldots$ & 4 & 2.0 & 44 & $\mathrm{~T}$ \\
DDO $125 \ldots \ldots \ldots \ldots$ & 0.6 & 3.8 & 19 & $\mathrm{~T}$ \\
DDO $13 \ldots \ldots \ldots \ldots$ & 1.6 & 3.1 & 30 & $\mathrm{H}$ \\
DDO $50 \ldots \ldots \ldots \ldots$ & 1.8 & 4.0 & 58 & $\mathrm{H}$ \\
\hline
\end{tabular}

${ }^{a}$ For explanation of column headings see $\S \mathrm{IV} a(\mathrm{i})$.

b T, Tully et al. 1978; H, Huchtmeier et al. 1981.

compared with known dwarf galaxies. Since we used the outer radius of the structures, i.e., the point at which disruption begins, the Holmberg radius should be the relevant length in the dwarf galaxy. In Table 2B, we tabulate masses, Holmberg radii, and velocity width for well-studied dwarf galaxies. These figures provide detailed support for the accretion of Magellanic type galaxies as the origin of the gas in NGC 2685. A further useful observation would be to find the metallicity of the associations seen in the rings to see if preprocessing is required.

The galaxy NGC 2685 is not unique, only the most outstanding and well-studied galaxy of its kind. Both NGC 1052 and NGC 2768 have similar properties (Illingworth 1981). In these galaxies, the stars are observed to revolve along the major axis; while there is a gaseous component evident in the [O II] line at $3727 \AA$ seen to revolve on the minor axis. Centaurus A (NGC 5128) has also had stellar motion detected on the major axis (Danziger et al. 1983), while the gas circulates in another plane. There are certainly other good candidates among the galaxies with dust lanes perpendicular to their major axes (Bertola and Galetta 1978; Kotanyi and Ekers 1978; Hawarden et al. 1981). In most of these systems, the relative concentration of the gas and stars makes it most likely that the origin of the gas is again due to the accretion of a small companion galaxy.

\section{ii) Dissipation in Triaxial Systems}

The physical phenomenon we are led to consider from the previous section is that of a satellite galaxy which is dragged inward by dynamical friction and then becomes disrupted and further evolves by dissipation. We consider first the dynamical friction.

Binney (1977) has calculated the effects of velocity anisotropies on dynamical friction by studying the evolution of massive particles in a spheroidal distribution of 2:1 flattening. The velocity dispersion in the plane of the massive particles is twice that out of the plane. He finds that the time scale for the anisotropy to be accentuated (i.e., the difference in the dynamical friction time scales in and out of the plane) is the same as the dynamical friction time scale in the plane. Binney considers this process to be of importance for the formation of linear structures in clusters of galaxies and for the destruction of globular clusters in the galactic disk.

The same exaggeration of velocity dispersion can be expected in triaxial galaxies. The separation of the Hamilton-Jacobi equations ( $(\mathrm{I} I a)$ is equivalent to the finding of principal velocity surfaces. Dynamical friction will act separately on the three principal velocities, though they will be coupled as the integrals of motion and hence the reduced potentials change. Thus a box orbit as it decays will be drawn to a radial orbit (a phenomenon which may have occurred in M31, if the linear features in the X-ray sources seen by van Speybroeck et al. [1979] are due to dynamical friction effects in a triaxial bulge [Stark 1977]). Any satellite which follows this course of evolution will disrupt at a few kpc on a highly eccentric orbit. The subsequent motion of the gas should be quite chaotic, with a substantial amount of gas finding its way to the central and perhaps even nuclear regions of the galaxy. If the original orbit is a tube orbit, the particle will be drawn closer to the 1-1 periodic orbit with only one nonzero action.

We imagine the gas in NGC 2685 as originating in this way. The satellite is drawn nearer to the elliptical 1-1 periodic orbit on the same time scale as it is drawn inward. The satellite is slowly disrupted and sheds gas into a sequence of tube orbits of diminishing radius. The gas then continues to circulate and settles closer to the periodic orbits as it dissipates. This process eventually leaves a cool, slightly oval disk with velocity dispersions 
corresponding to a gas temperature of $10^{4} \mathrm{~K}$. In NGC 2685 this seems to have occurred in both of the possible configurations for tube orbits, implying a potential which is triaxial rather than oblate or prolate. In NGC 2768, gas is circulating about the long axis as determined by observations of the $3727 \AA$ line by Illingworth (1981).

This discussion can be made more rigorous by the use of results in § II and a method due to Mel'nikov (1963; for a more complete discussion see Holmes 1979). Mel'nikov's method considers the evolution of dissipational systems which are close to Hamiltonian. Stable fixed points (points which are transformed to themselves by the time evolution operator) become the sinks or attractors for nearby regions of phase space. When we linearize about the stable 1-1 orbits, we find stable fixed points in the reduced potentials. These are the attractors in the reduced space. Since the dissipation also acts to diminish the amplitude of the periodic motion (albeit at a lesser rate), the location of the fixed points is not actually fixed but moves slowly. The result is that heuristically described above.

In this scenario, many of the unusual gaseous rings may be young features, having resulted from the recent capture of a small companion galaxy. In this case they may not have fully settled and could display warping, as observed in the outer parts of spiral galaxies (Sancisi 1976; Tubbs and Sanders 1979). If the features are indeed young and unusual, is there any need to postulate triaxiality and the existence of stable orbit configurations? The alternative is a nearly polar capture by an oblate object with long precessional times allowing the observed unusual objects. There are different predictions in the two cases. These are shown in Figure 3. In the triaxial case, the shorter precession and dynamical times in the central region led to a smoother and more "polar" orbit. Thus a bending toward the short axis as the center is approached is expected. In the other case, the reverse is predicted. The only observed example which comes to mind is NGC 5128 (Centaurus A) where the gas is found in the configuration expected in the triaxial picture.

The process by which the tube orbits slowly settle to the plane is straightforward and enables "age-dating" of the configuration to be made. The physics is similar to that of orbits in an axisymmetric potential. In the axisymmetric case we expect a ring of gas to evolve via differential precession damped by cloud-cloud collisions. The result is a disk in the fundamental plane of symmetry. This process occurs on the time scale of the differential precession of the orbits. This time scale is the dynamical time divided by the eccentricity of the potential $\left(t_{\mathrm{dyn}} / \epsilon\right)$. For an E3 galaxy with a rotation velocity of $300 \mathrm{~km} \mathrm{~s}^{-1}$, this time scale is $10^{9}$ years at $5 \mathrm{kpc}$. This implies an age of $\sim 5 \times 10^{8}$ years in the case of Cen A (see also Tubbs 1980).
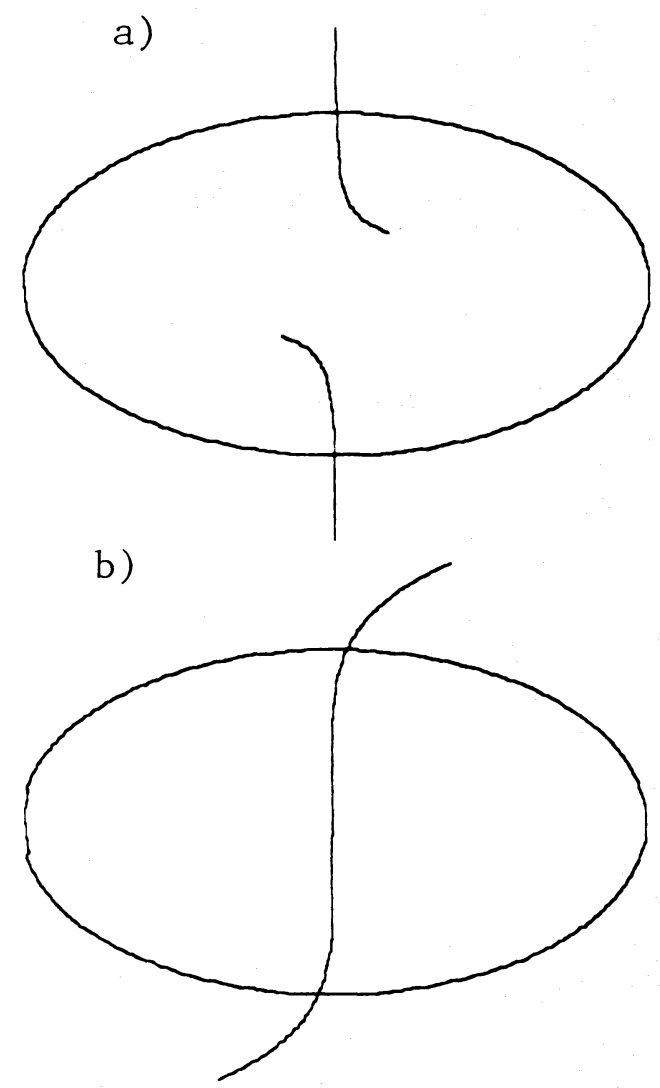

Fig. 3.-Appearance of a dust lane (open curve) relative to the stellar figure (ellipse) for system formed by (a) capture in the unstable polar orbit of an oblate figure, $(b)$ capture in the stable periodic orbit of a triaxial figure. 
Van Albada, Kotanyi, and Schwarzschild (1982) have proposed a similar model for the existence of layers of dust and gas in "unusual" configurations. They attempted to explain the bending of the layers by placing gas in retrograde orbits in a rotating figure. They further used this model to explain the bending of radio jets. Several comments are in order. Their model applied to Cen A requires the jet velocity to be comparable to the velocity dispersion of the galaxy. In such a situation, buoyancy effects are so pronounced as to render a discussion of bending moot. This scenario also requires excessive flow rates. In order to account for the energy contained in the radio lobes of Cen A, $\sim 10^{12} M_{\odot}$ would have to have been expelled. There are other specific observational checks: (1) the orbits must be retrograde (current indications are that they are direct, cf. Danziger et al. 1983); and (2) the cooling time is shorter than the time for transient bends to disappear, so the velocity dispersion of the gas is $10-20 \mathrm{~km} \mathrm{~s}^{-1}$.

The behavior of gas near unstable periodic orbits is another interesting problem. Under the right conditions, these orbits, together with the action of dissipation, can lead to "repellors" or the phenomenon of "strange attraction" (Ott 1981). The unstable elliptical orbit about the intermediate axis behaves like the polar orbits in an axisymmetric potential and is a "repellor." We have not found "strange attractors" in the potentials we have examined. Whether "repellors" or "strange attractors," the unstable orbits do not act as sinks or normal attractors and there is not any justification for associating such phenomena as warped disks with these orbits (Binney 1978).

\section{b) Gas Motions. II. Central Regions}

In this section, we investigate the properties of triaxial potentials relevant to observations and inferred physical processes in the galactic center and in active galactic nuclei. Once again, we use the property that the stable, noninteresting elliptical orbits behave like dynamical attractors (Chillingworth 1976), so that under the action of dissipation the gas will settle into these orbits. The features we will consider are: (1) the structure of the elliptical orbits at energy just greater than the bifurcation energy where they emerge, (2) velocity fields which mimic that of a supermassive object at the center, (3) the existence of stable elliptical orbits which are tilted with respect to all the symmetry planes, a possible explanation for the tilted disk observed at the Galactic center, (4) the possibility of enhanced fueling due to the disappearance of all angular momentum integrals and hence the absence of any loss-cone (Frank and Rees 1976), (5) the effect of "symmetry restoration" due to the growth of an axisymmetric central massive object.

i) Structure of the Elliptical Orbits and Central Velocity Fields

In $\S$ III $b$, we calculated the critical energy at which the first elliptical orbits bifurcate from the radial orbits along the shorter axis. The first of these orbits is highly elongated, though they quickly circularize. We have explicitly calculated the closed elliptical orbits in the model potential of Schwarzschild (eqs. [3], [4]), and these are shown in Figure 4. Here $X$ is the longest axis and $Y$ is the intermediate axis (the orbits are calculated in the plane $z=0$ ). Note that the orbits counteralign to the figure; i.e., their long axis is the $Y$-axis. At low amplitude the spring constant in the $Y$ direction is stiffer than that in $X$. At larger amplitudes of oscillation in $Y$, the nonlinearity of the

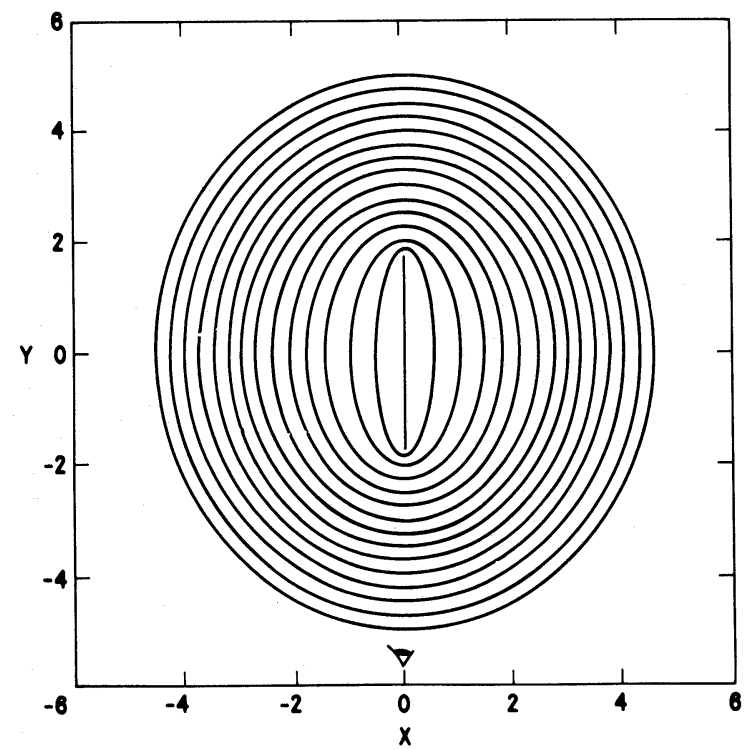

FIG. 4.-Closed elliptical orbits in the model proposed by Schwarzschild 
potential allows locking of the $X$ and $Y$ frequencies. At this point the $Y$-axial orbit becomes unstable and the elliptical orbits emerge at the bifurcation point.

From Figure 4, one may see that the changing eccentricity of the orbits can produce interesting effects on the observed rotation curve. From the perspective of the "eye" in Figure 4, it is clear that the observed velocity is not equal to zero at the center. Figure 5 shows the rotation curve seen by the observer depicted in Figure 4 together with the symmetrized rotation curve. Note the central rise and unusually rapid declines which are possible. Such features in the past have been assumed to be due to supermassive objects (Lacy et al. 1980) and disks with holes producing a ring which tugs outward (Caldwell and Ostriker 1982). This latter explanation is probably not possible in any case. If the rotation really declined faster than Keplerian, the epicyclic frequency would be imaginary; i.e., there would be a kinematic instability of the type first discovered by Lord Rayleigh. We should instead take such features to be sure indicators of noncircular motions due to nonaxisymmetric potential fields.

\section{ii) Tilted Disks}

In the innermost $600 \mathrm{pc}$ of the Galaxy, Liszt and Burton (1980) report that the disk is tilted at an angle of $\sim 22^{\circ}$ with respect to the plane defined by the disk at larger radii. Clearly this configuration is unstable in an axisymmetric potential. We ask whether such a configuration might exist and be stable in a potential which is not axisymmetric, much in the same way that we modeled the unusual state of gas in the Spindle galaxy.

First, we note that several authors (van Albada, Kotanyi, and Schwarzschild 1982; Tohline and Durisen 1982) have found a family of retrograde orbits which are tilted by the Coriolis force in a rotating triaxial potential. Since the disk in the galactic center is direct, their orbits are not relevant there.

In $\S$ III $c$, we analyzed the existence and stability of 1-1-1 resonant orbits in a potential with harmonic plus quartic terms. Here we are interested in the Type IV orbits with one oscillation out of phase and with the other two producing an inclined elliptical orbit. The stability conditions for these orbits are given in equations (20)-(23).

The problem with this approach is that all the Type IV orbits in the potential (8) occur at an energy at which the particles are barely bound (or even just unbound). It does, however, tell us where to look and gives some believable general results. For example, it predicts that the only one of these orbits which is stable is that which has the oscillation along the intermediate axis out of phase with the other two. Further, they do not exist in potentials like that used by Schwarzschild (eqs. [3], [4]) even when different axial ratios are allowed. This is particularly unfortunate, as, had they existed, it would have given us an intermediate energy regime that had been explored by others.

The aforementioned drawbacks aside, it is straightforward to find stable tilted orbits by varying the structure constants in the potential (8). One example of such a tilted orbit is shown in Figure 6 . The quoted values of the structure constants (see legend to Fig. 6) have no particular significance.

The orbit in Figure 6 was found and calculated as follows. The structure constants were chosen subject to the constraint imposed by equation (23a), and an approximation to the orbit was calculated using the Galerkin method $(\S \mathrm{III} b)$. If this low-order approximation satisfied equation (23b), the orbit was calculated numerically for 10-20 dynamical times from starting conditions given by the Galerkin results. Thus the starting conditions are only close to

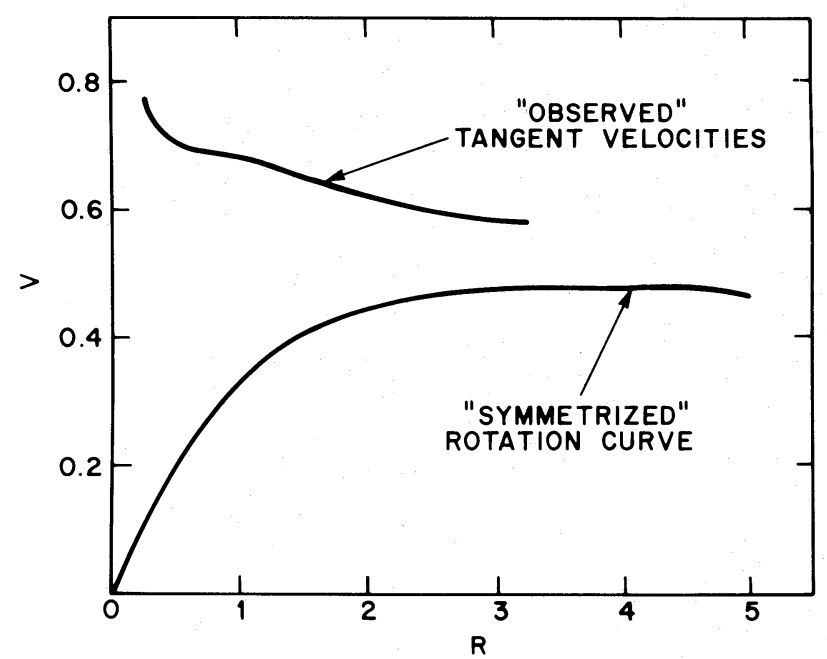

FIG. 5.--Rotation curves for Schwarzschild's model. Top curve is found by sighting through the orbits shown in Fig. 4. Bottom curve is given by $\left[0.5\left(F_{x}+F_{y}\right) r\right]^{1 / 2}$, where $F_{x}, F_{y}$ are the forces on the $X$ and $Y$ axes at a distance $r$ from the center. 

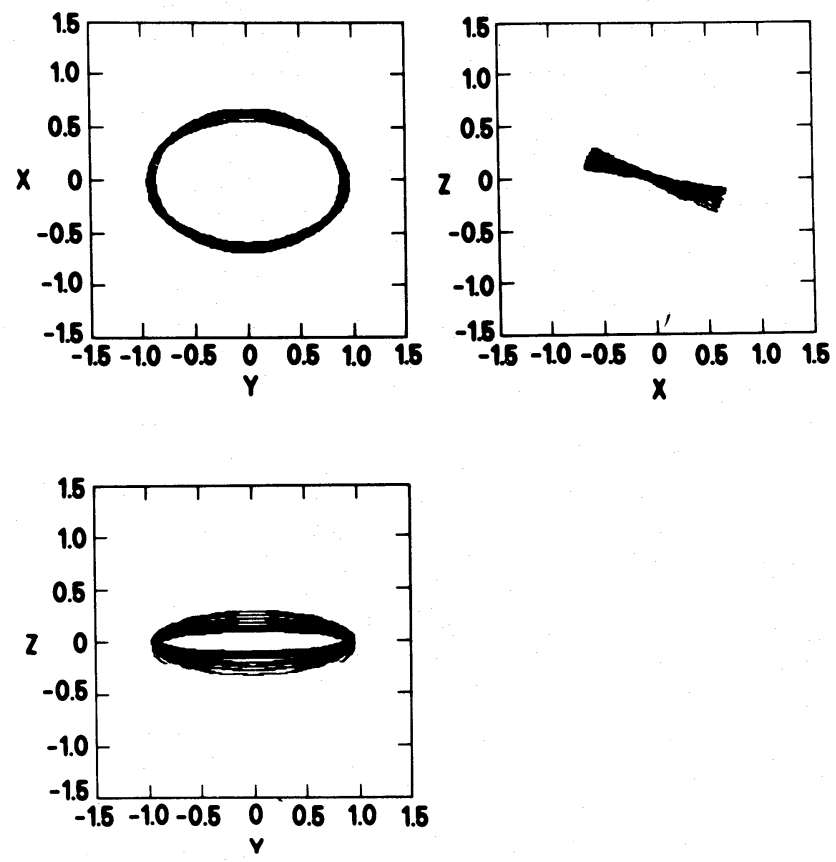

FIG. 6.-Projection of the trajectory of a tilted orbit onto the three planes of symmetry of the potential. The structure constants (see eq. [8]) are: $a=0.24, b=0.31, c=0.16, d=0.11, e=0.21, f=0.45, g=0.48, h=0.53, k=0.85$.

the periodic orbit; and if the calculated orbit seems to oscillate about the periodic orbit (rather than precess to one of the planes), we conclude that we have found an inclined elliptical tube orbit.

Two other general features emerged in the three cases (i.e., different set of structure constants) which were calculated. The orbits had a tilt angle (as shown in the $X-Z$ plane of Fig. 6) which was constant over the range of energy (radius) examined, and their eccentricity (as shown in the $X-Y$ plane of Fig. 6) increased with increasing energy (radius). These features were proposed by Liszt and Burton (1980) in their modeling of the kinematics in the galactic center.

Constructing detailed models of the galactic center is beyond the scope of the present paper and would seem premature from an observational standpoint. Heiligman (1982) has modeled his observations of velocity field with orbits calculated in the symmetry plane of a Schwarzschild model. His model is significant as it is the first dynamical model which accounts for the large radial motions. Liszt and Burton (private communication) have discovered a more extensive and massive disk inclined at roughly $90^{\circ}$ with respect to the disk reported in their previous work. The rotation of the underlying figure will undoubtedly play a prominent role in the dynamics of both these disks. Recently Brown (1982) has proposed the existence of a precessing jet to fit the kinematics of the clouds observed in hydrogen recombination lines. If the tilted disk is locked into the rotation of the figure, jet precession may follow. Van Albada, Kotanyi, and Schwarzschild (1982) have proposed using the large-scale tumbling of a galaxy to do this. The problems of buoyancy and flow rates were described previously (§ IV $a[\mathrm{ii}]$ ). For less energetic flows and/or the faster tumbling of a more compact component, such a mechanism might be operative. A significant difference is that the disk configuration proposed here can have direct motion (i.e., need not involve retrograde orbits). Data are emerging rapidly and will hopefully allow more quantitative comparisons in the near future.

\section{iii) Enhanced Fueling Rates in Active Galaxies}

The main problem in supplying material to a nuclear source is shedding the angular momentum, thus reducing or eliminating any centripetal barrier. In the past, axisymmetry has been assumed and two basic alternatives for the fuel, i.e., stars and gas, have been considered. Gas reaches the center by the action of viscous and magnetic torques which serve to transport angular momentum outward and matter inward. These processes are effective in the nuclear accretion disk, but the supply into the center may pose problems (Gunn 1979). Stars are consumed by tidal capture. The key issue here is that only stars with extremely low values of the angular momentum are available for capture. These form the loss cone (Frank and Rees 1976) and, when depleted, the fuel is spent.

In a nonaxisymmetric potential, the situation is certainly different, as the angular momentum of an individual particle is no longer a conserved quantity. Again, we consider separately gas and stars as the possible fuel. In Schwarzschild's (1979) model, over $80 \%$ of the stars are in box orbits. Each of these has a nonvanishing probability 
amplitude at the center. Clearly, the usual loss-cone problem is gone (Norman and Silk 1983). If the material is in the form of gas, the effect of any viscosity is amplified in the region where the eccentricity of the elliptical orbits is changing rapidly. This will enable gas to fall into the inner region from a distance of a few core radii. To illustrate this, Figure 7 shows the elliptical orbit launched at a radius of 1.8 core radii (solid line) which is perturbed by an amount corresponding to a velocity of $14 \mathrm{~km} \mathrm{~s}^{-1}$ on a mean rotation velocity of $100 \mathrm{~km} \mathrm{~s}$ (dots indicate the trajectory of this particle). This illustrates the sensitivity to initial conditions of these inner orbits and the ease with which clouds may be perturbed into orbits which reach the center. G. Heiligman (private communication) believes that this effect is critical to an understanding of the outermost contours of his longitudevelocity map of the galactic center. The resultant shocks may also lead to the observed excited gas with large line widths. Note that although rotation is the ultimate cause of these widths, no correlation between the orientation of a galaxy and observed line width should be evident (Woltjer 1959; Simkin, Su, and Schwarz 1980). We also note the tendency of the cloudlets to pile up at the ends of the unperturbed orbit. This region of enhanced density and low velocity may be a key to the enhanced star formation observed at the ends of many bars in barred spiral galaxies.

A question which arises is: How does one stem the flow? One possibility would seem to be that the growth of a central massive object might lead to symmetry restoration, thus eliminating box orbits and stabilizing tube orbits. In fact, the latter occurs, while the former does not.

To examine the effect of a central massive object on the elliptical orbits, in Figure 8 the orbits of Figure 4 have been recomputed with the addition of a central mass point, with the mass of the central object varying from $0.025 M_{c}$ to $0.5 M_{c} .\left(M_{c}\right.$ is the symmetrized core mass, defined in a manner analogous to the symmetrized rotation curve of Fig. 5.) Even with a point mass as small as $0.025 M_{c}$ the bifurcation of axial to elliptical orbits has vanished. Although the changing ellipticity of the orbits will enhance the effects of dispersions in the velocities and viscosity, this effect is also suppressed by the time that the point mass is half that of the core. This leads to the attractive hypothesis that the growth of the central massive object is responsible for its own demise.

The changes observed in box orbits are less interesting, as the growth of the point mass only further elongates the box. A simple explanation of this phenomenon lies in the ability to generate box orbits by "dropping" particles at zero velocity anywhere in the potential. In an axisymmetric potential this produces a linear orbit which goes through the center. By increasing the mass of the central object, this limiting case is smoothly approached.

\section{CONCLUSIONS}

We have presented a variety of analytic means for examining the existence and stability of orbit families in triaxial potentials which approximate the potential fields of triaxial galaxies. These analytical methods seem most fruitful when applied to the fate of gas in these systems.

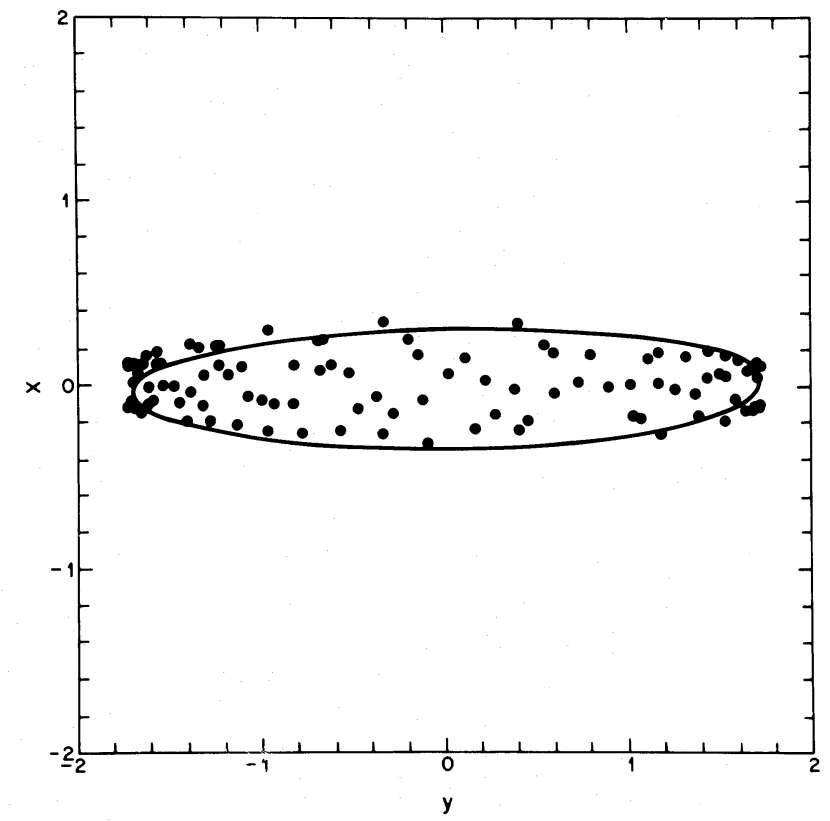

Fig. 7.-Solid line, the closed elliptical orbit which crosses the $X$ axis at $Y=1.8$. Dots, points on the trajectory of an orbit perturbed from the elliptical orbit by launching the orbit from $X=0, Y=1.8$ with the same velocity as before, but at an angle difference of $5^{\circ}$. 


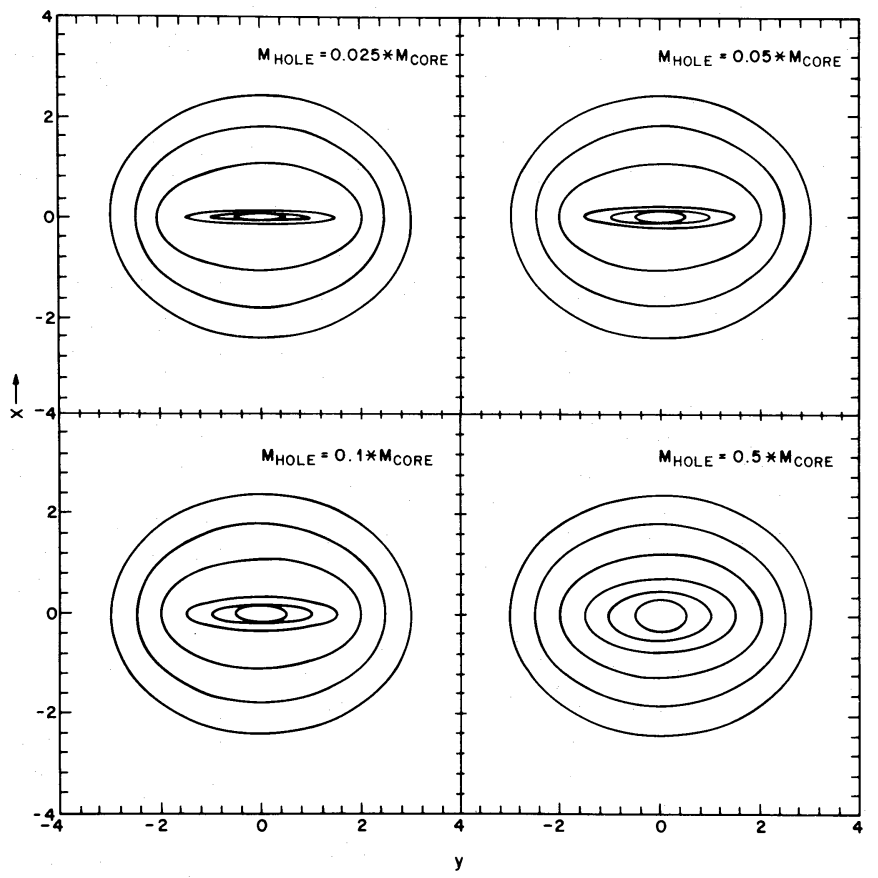

FIG. 8.-Orbits calculated as in Fig. 4 with the addition of a central point mass $\left(M_{\text {hole }}\right)$ given in units of the core mass $\left(M_{\text {core }}\right)$. Note the absence of any bifurcation in even the $M_{\text {hole }}=0.025 M_{\text {core }}$ case.

As important highlights of our analysis we point to (1) the recognition that triaxial galaxies may possess two stable disks inclined at right angles, as is seen in the Spindle galaxy, NGC 2685. (2) The features seen in the Spindle are compatible with the accretion of dwarf galaxies with properties like those of the dwarfs in the Local Group. (3) Various scenarios for inclined disks inside elliptical galaxies may be tested by examining their photometric and kinematical structure. In particular, if the disks are due to attraction to stable orbits, the inner parts are expected to align closer to the axis than the outer parts, whereas unstable orbits in oblate galaxies should show precession to the plane (see Fig. 3). Current observations favor the former. (4) The effect of anisotropic dynamical friction may lead to linear features as seen in M31 (van Speybroeck et al. 1979). (5) Rising velocity fields observed in the central regions of galaxies must be interpreted with caution. (6) Stable orbits tilted with respect to the symmetry axis may exist and provide a dynamical explanation for the tilted disk seen in our Galaxy. (7) Gas motion into the center is enhanced and may be shunted by the growth of a central massive object.

We thank W. B. Burton, T. deZeeuw, G. Heiligman, H. Liszt, D. Merritt, A. Penzias, M. Schwarzschild, W. Shane, and J. A. Tyson for lively discussions, often of data in advance of publication. Critical readings of the manuscript by Ken Jacobs and an anonymous referee have greatly helped the presentation. This work was started while G. L. enjoyed the hospitality of the Institute of Astronomy, Cambridge, and the support of a NATO postdoctoral fellowship.

\section{APPENDIX \\ ACTIONS, ANGLES, AND ORBIT DENSITIES FOR THE POTENTIAL OF § II}

In this section the actions and frequencies are derived for the potential of equation (1). The orbits are integrated, and the configuration space density of the orbit averaged over time is derived.

The equation of radial motion is the same as that analyzed by Eggen, Lynden-Bell, and Sandage (1962). The radial action is given by

$$
J_{r}=\frac{1}{2 \pi} \oint P_{r} d r=\frac{1}{2 \pi} \oint\left[H-\frac{I_{3}}{r^{2}}+\xi(r)\right]^{1 / 2} d r=G M(-2 H)^{-1 / 2}-\frac{1}{2}\left[I_{3}+\left(I_{3}{ }^{2}+4 G M b\right)^{1 / 2}\right],
$$


with the corresponding frequency

$$
\omega_{r}=\left.\frac{\partial H}{\partial J_{r}}\right|_{J_{\theta}, J_{\phi}}=\frac{(-2 H)^{3 / 2}}{G M} .
$$

The azimuthal $(\phi)$ action in the case where $\phi$ is periodic with period $2 \pi$ is given by

$$
J_{\phi}=\frac{1}{2 \pi} \oint P_{\phi} d \phi=\frac{1}{2 \pi} \oint\left[I_{2}+\beta \cos ^{2} \phi\right]^{1 / 2} d \phi=2 \frac{\left(I_{2}\right)^{1 / 2}}{\pi} E\left(\frac{2 \beta}{I_{2}}\right),
$$

where

$$
E\left(m_{1}\right)=\int_{0}^{\pi / 2}\left(1-m_{1} \sin ^{2} \theta\right)^{1 / 2} d \theta
$$

is the complete elliptic integral of the second kind. If $\phi$ librates between $\pm \arcsin \left[\left(I_{2}+\beta\right) / 2 \beta\right]^{1 / 2}$, the $\phi$ action is given by

$$
J_{\phi}=\frac{2\left(I_{2}+\beta\right)^{1 / 2}}{\pi} F\left[\arcsin \left(\frac{I_{2}+\beta}{2 \beta}\right)^{1 / 2},\left(\frac{I_{2}+\beta}{2 \beta}\right)\right]
$$

where $F(\phi, m)$ is an elliptic integral of the first kind (Abramowitz and Stegun 1964). The corresponding frequency is:

$$
\omega_{\phi}=\left(\frac{\partial H}{\partial I_{3}}\right)\left(\frac{\partial \ln \Omega_{1}}{\partial I_{3}}\right) \frac{\pi}{2}\left(I_{2}\right)^{1 / 2} \times \begin{cases}\frac{1}{K\left[\left(2 \beta / I_{2}\right)^{1 / 2}\right]} & \text { for periodic motion } \\ \frac{1}{F\left[\arcsin \left(I_{2} / 2 \beta^{1 / 2}, 2 \beta / I_{2}\right)\right]} & \text { for librating motion }\end{cases}
$$

where

$$
K(x)=\int_{0}^{\pi / 2}\left(1-x \sin ^{2} \theta\right)^{-1 / 2} d \theta
$$

is the complete elliptic integral of the first kind.

$$
\begin{gathered}
\Omega_{1}=\frac{2}{\pi(\alpha)^{1 / 2}} \int_{c_{1}}^{c_{2}} \frac{d x}{\left(x^{2}-d\right)\left(x^{2}-e\right)} \\
d, e=2 \alpha+I_{3} \pm\left[\left(2 \alpha+I_{3}\right)^{2}-\alpha\left(I_{3}-I_{2}+\alpha\right)\right]^{1 / 2}
\end{gathered}
$$

and $c_{1}, c_{2} \in[d, e, 1]$ depending on the limits of libration.

The $\theta$ action is:

$$
J_{\theta}=\frac{1}{2 \pi} \oint P_{\theta} d \theta=\frac{1}{2 \pi} \oint\left(I_{3}-\frac{I_{2}}{\sin ^{2} \theta}+\alpha \sin ^{2} \theta\right)^{1 / 2} d \theta=\frac{1}{\pi}\left\{a^{1 / 2} J^{1}+\left(\frac{I_{3}^{2}}{\alpha}\right)^{1 / 2} J^{0}-\left(\frac{I_{2}{ }^{2}}{\alpha}\right)^{1 / 2} J^{-1}\right\}
$$

where

$$
J^{n}=\int_{c_{1}}^{c_{2}} \frac{t^{n} d t}{[(1-t)(a-t)(b-t)]^{1 / 2}}
$$

Here,

$$
a, b=\frac{-I_{3} \pm\left(I_{3}^{2}+4 \alpha I_{2}\right)^{1 / 2}}{2 \alpha}
$$

and $c_{1} c_{2} \in[a, b, 1]$ depending on the specific libration limits. The $J^{n}$ are tabulated in Gradsteyn and Ryzshik (1980), $\$ 3.13$ and 3.14 , and the various cases are generally linear combinations of elliptic integrals. The corresponding frequency is:

$$
\omega_{\theta}=\frac{\partial H}{\partial J_{\theta}}=\frac{\partial H}{\partial I_{3}} \frac{\partial I_{3}}{\partial J_{\theta}}
$$

and

$$
\left(\frac{\partial I_{3}}{\partial J_{\theta}}\right)^{-1}=\frac{1}{2 \pi} \oint \frac{d \theta}{\left[I_{3}\left(I_{2} / \sin ^{2} \theta\right)+\alpha \sin ^{2} \theta\right]^{1 / 2}}=\frac{2}{\alpha^{1 / 2} \pi a} \operatorname{sn}^{-1}\left(\frac{c}{b}, \frac{b^{2}}{a^{2}}\right)
$$


where $\mathrm{sn}^{-1}$ is an inverse Jacobian elliptic function and the $\theta$ motion librates between $\pm \arccos c$ (Gradsteyn and Ryzshik, § 17.4.41) and

$$
a^{2}, b^{2}=\frac{1}{2}\left(\frac{2 \alpha+I_{3}}{\alpha}\right)\left[1 \mp 4 \frac{\left(I_{3}-I_{2}+\alpha\right)}{\left(2 \alpha+I_{3}\right)}\right]^{1 / 2} .
$$

The configuration space density of an orbit averaged over time is easily derived. As always this density is the integral of the phase space distribution function integrated over momenta, i.e.,

$$
\rho=\int d^{3} p f(\boldsymbol{p}, \boldsymbol{x}),
$$

so that the density of a single orbit is:

$$
\begin{aligned}
\rho_{\text {orb }} & =\int d^{3} p \delta(H-h) \delta\left(I_{2}-i_{2}\right) \delta\left(I_{3}-i_{3}\right)=\frac{\partial\left(P_{r}, P_{\theta}, P_{\phi}\right)}{\partial\left(I_{1}, I_{2}, I_{3}\right)} \\
& =\left[I_{1}-\frac{I_{3}}{r^{2}}+\xi(r)\right]^{-1 / 2}\left[I_{3}-\frac{I_{2}}{\sin ^{2} \theta}+\eta(\theta)\right]^{-1 / 2}\left[I_{2}+K(\phi)\right]^{1 / 2} .
\end{aligned}
$$

Calculating orbit densities in this manner has several possible uses. If only two integrals had been known, a comparison of the density calculated in this manner with that found by numerical integration of the orbit would demonstrate the existence of a third integral of motion without the need for surfaces of section (Lake 1979). Scaling properties in self-similar models may also be calculated in this manner. Finally it provides a method of solution to the PoissonVlasov equations by analytical calculation of particle orbit densities which may be summed with linear programming techniques or polynomial expansions to recreate the assumed density (Lake 1979). This method combines features of the methods of Lynden-Bell (1962) and Schwarzschild (1979).

\section{REFERENCES}

Aarseth, S. J., and Binney, J. J. 1978, M.N.R.A.S., 185, 227.

Abramowitz, M., and Stegun, I. A. 1964, Handbook of Mathematical

Functions (Washington: National Bureau of Standards).

Arnold, V. I. 1963, Russian Math Surveys, 18, 85.

-1978, Mathematical Methods of Classical Mechanics (New York: Springer-Verlag).

Arp, H. 1967, Ap. J. Suppl., 14, 1.

Berry, M. 1978, in Topics in Nonlinear Dynamics A Tribute to Sir Edward Bullard, ed. S. Jorna (New York: American Institute of Physics).

Bertola, F., and Galletta, G. 1978, Ap. J. (Letters), 226, L115.

Binney, J. J. 1977, M.N.R.A.S., 181, 735.

- 1978, M.N.R.A.S., 183, 501 1981, M.N.R.A.S., 196, 455

Brown, R. L. 1982, preprint.

Caldwell, J., and Ostriker, J. P. 1981, Ap. J., 251, 61.

Chillingworth, D. R. J. 1976, Differential Topology With a View tó Applications (London: Pitman Press).

Chirikov, B. V. 1979, Phys. Rept., 52, 263.

Danziger, J., Davies, R., Jones, J., and Jones, B. J. T. 1983, in preparation.

Dell'Aqua, F. A. 1908, Math. Ann., 66, 394.

de Zeeuw, T. 1982, preprint.

de Zeeuw, T., and Merritt, D. 1983, Ap. J., 267, 571

Einstein, A. 1917, Verh. Dt. Phys. Ges., 19, 82.

Eggen, O., Lynden-Bell, D., and Sandage, A. 1962, Ap. J., 136, 748.

Evans, D. 1952, M.N.R.A.S., 112, 606.

Frank, J., and Rees, M. J. 1976, M.N.R.A.S., 176, 633.

Galerkin, B. G. 1915, Series Developments for some Cases of Equilibrium of Plates and Beams (in Russian) (Petrograd: Wiestnik Ingenerow).

Goodman, and Schwarzschild, M. 1980, Ap. J., 245, 1087.

Gradsteyn, I. S., and Ryzshik, I. M. 1980, Table of Integrals Series and Products (New York: Academic Press).

Gunn, J. E. 1979, in Active Galactic Nuclei, ed. C. Hazard and S. Mitton (Cambridge: Cambridge University Press).

Hall, G., and Watt, J. M. 1976, Modern Numerical Methods for Ordinary Differential Equations (Oxford: Clarendon Press).

Hawarden, T. G., Elson, R. A. W., Longmore, A. J., Tritton, S. B., and Corwin, H. G. 1981, preprint.
Heilegman, G. 1982, in preparation.

Heilegman, G., and Schwarzschild, M. 1979, Ap. J., 233, 872.

Helleman, R. H. G., and Bountis, T. 1977, in Stochastic Behavior in Hamiltonian Systems, ed. G. Casati and J. Ford (New York: Springer-Verlag).

Hénon, M., and Heiles, C. 1964, A.J., 69, 73.

Holmberg, E. 1969, Ark. für Astr., 5, 305.

Holmes, P. 1979, Phil. Trans. Roy. Soc. London, 292, 419

Huchtmeier, W. K., Seiradakis, J. H., and Materne, J. 1981, Astr. Ap., $102,134$.

Illingworth, G. 1977, Ap. J. (Letters), 218, L43.

. 1981, lecture at CECAM workshop, Paris.

King, I. R. 1978, Ap. J., 222, 1.

Kolmogorov, A. N. 1954, Dokl. Akad. Nauk, USSR, 98, 527 (English Transl. Los Alamos Scientific Laboratory, LA-TR-71-67).

Kotanyi, C. G., and Ekers, R. D. 1978, Astr. Ap., 73, L1.

Lacy, J. H., Baas, F., Towers, C. H., and Geballe, T. R. 1980, Ap. J. (Letters), 227, L17.

Lake, G. 1979, Princeton University thesis.

- 1981, Ap. J., 243, 111.

1982, lecture at APS Plasma Physics meeting, New Orleans.

Lake, G., and Norman, C. 1982, in The Galactic Center, ed. G. R. Reigler and R. D. Blandford (New York: American Institute of Physics).

Levi-Cevita, T. 1904, Math. Ann., 59, 383.

Liszt, H., and Burton, W. B. 1980, Ap. J., 236, 779.

Lynden-Bell, D. 1962a, M.N.R.A.S., 123, 447.

- 1962b, M.N.R.A.S., 124, 95.

Mel'nikov, V. K. 1963, Trans. Moscow Math. Soc., 12, 1.

Miller, R. H. 1978, Ap. J., 224, 32.

Miller, R. H., and Smith, B. F. 1980, Ap. J., 235, 793

Moser, J. 1962, Machr. Ges. Wiss. Göttingen Math.-Phys. Kl., 29, No. $1,1$.

Norman, C., and Silk, J. 1983, Ap. J., 263, in press.

Ostriker, J. P., and Turner, E. L. 1979, Ap. J., 234, 785.

Ott, E. 1981, Rev. Mod. Phys., 53, 655.

Percival, I. C. 1973, J. Phys. B, 6, L229.

Prendergast, K., and Tomer, E. 1970, A.J., 75, 674.

Richstone, D. 1980, Ap. J., 234, 825. 
Rosenbluth, M. N., Sagdeev, R. Z., Taylor, J. B., and Zaslavsky, G. M. 1966, Nucl. Fusion, 6, 297.

Sancisi, R. 1976, Astr. Ap., 53, 159.

Schecter, P. L., and Gunn, J. E. 1978, A.J., 83, 1360.

Schwarzschild, M. 1979, Ap. J., 232, 236.

Shane, W. W. 1980, Astr. Ap., 82, 314.

Siegel, C. L. 1954, Math. Ann., 128, 144.

Simkin, S. M., Su, H. J., and Schwarz, M. P. 1980, Ap. J., 237, 404.

Stäckel, P. 1890, Math. Ann., 35, 91

Stark, A. A. 1977, Ap. J., 213, 368.

Tohline, J. T., and Durisen, R. H. 1982, Ap. J., $257,94$.

Toomre, A. 1977, in The Evolution of Galaxies and Stellar Populations,

ed. B. M. Tinsley and R. B. Larson (New Haven: Yale University Observatory).

Tubbs, A. D. 1980, Ap. J., 241, 969.

Tubbs, A. D., and Sanders, R. H. 1979, Ap. J., 230, 736.

Tully, R. B., Bottinelli, L., Fisher, J. R., Goughenheim, L., Sancisi, R., and van Woerden, H. 1978, Astr. Ap., 63, 37.
Ulrich, M-H. 1975, Pub. A.S.P., 87, 965.

Urabe, M. 1966, in Numerical Solutions of Nonlinear Differential Equations, ed. D. Greenspan (New York: Wiley).

Urabe, M., Yanagiwara, H., and Shinohara, Y. 1960, J. Sci. Hirosh., 23, 325 .

van Albada, T. S. 1982, M.N.R.A.S., in press.

van Albada, T. S., Kotanyi, C. G., and Schwarzschild, M. 1982, M.N.R.A.S., 198, 303.

van der Burgh, A. H. P. 1976, J. Sound Vibr., 49, 93.

van Speybroeck, L., Epstein, A., Forman, W., Giacconi, R., Jones, C., Liller, W., and Smarr, L. 1979, Ap. J. (Letters), 234, L45.

Verhulst, F. 1979, Phil. Trans. Roy. Soc. London, 290, 435.

Weinacht, J. 1928, Math. Ann., 91, 279.

Wilkinson, A., and James, R. 1982, M.N.R.A.S., 199, 171.

Wilson, C. P. 1975, A.J., 80, 175

Woltjer, L. 1959, Ap. J., 130, 138.

GeORGE LAKE: 1D-236, Bell Laboratories, Murray Hill, NJ 07974

Colin Norman: Institute of Astronomy, Madingley Road, Cambridge, CB3 0HA, England 